\title{
The role of empathy in the neural responses to observed human social touch
}

\author{
Leehe Peled-Avron ${ }^{1}$ • Einat Levy-Gigi ${ }^{2}$ - Gal Richter-Levin ${ }^{2}$. \\ Nachshon Korem ${ }^{1}$ • Simone G. Shamay-Tsoory ${ }^{1}$
}

Published online: 10 May 2016

(C) Psychonomic Society, Inc. 2016

\begin{abstract}
One of the ways in which individuals convey feelings and thoughts to one another is through touch. Although the neural responses to felt and observed tactile stimuli between an inanimate object and a part of the human body have been vastly explored, the neural responses to observed human interaction involving touch are not well understood. Considering that the observation of social touch involves vicarious sharing of emotions, we hypothesized that levels of empathic traits modulate the neural responses to observed touch and focused on the attenuation in the mulalpha rhythm $(8-13 \mathrm{~Hz})$, a neural marker that has been related to sensorimotor resonance. Fifty-four participants observed photos depicting social touch, nonsocial touch, or no touch while their electroencephalography (EEG) activity was recorded. Results showed that interindividual differences in levels of empathic traits modulated both behavioral and electrophysiological responses to human social touch, such that highly empathic participants evaluated human social touch as inducing more pleasant emotions and exhibited greater mu suppression upon observation of human social touch compared to less empathic participants. Specifically, both the behavioral and the electrophysiological responses to observed social touch were predicted by levels of personal distress, a measure of emotional contagion. These findings indicate that the behavioral and electrophysiological responses to observed social touch are modulated by levels of empathy.
\end{abstract}

Leehe Peled-Avron

leehee.peled@gmail.com

1 Psychology Department, University of Haifa, Mount Carmel, Haifa 31905, Israel

2 The Institute for the Study of Affective Neuroscience, University of Haifa, Haifa, Israel
Keywords Empathy $\cdot$ Mu rhythm $\cdot$ EEG $\cdot$ Interpersonal touch $\cdot$ Somatosensory

An accumulating body of evidence suggests that tactile stimuli and observed tactile stimuli activate the same core brain networks, namely the somatosensory cortices (Bolognini, Rossetti, Convento, \& Vallar, 2013; Keysers et al., 2004; Rizzolatti, Fogassi, \& Gallese, 2001; Rossetti, Miniussi, Maravita, \& Bolognini, 2012; Schaefer, Heinze, \& Rotte, 2005, 2012; Schaefer, Xu, Flor, \& Cohen, 2009). Although previous studies have examined the mechanisms underlying tactile stimuli comprising a human body part (hand, head, neck, arm, leg) and an inanimate object (Blakemore, Bristow, Bird, Frith, \& Ward, 2005; Bufalari, Aprile, Avenanti, Di Russo, \& Aglioti, 2007; Coll, Bird, Catmur, \& Press, 2015; Lamm, Silani, \& Singer, 2015), the mechanisms underlying observed social touch are still largely unknown.

One of the ways in which individuals convey feelings and thoughts to one another is through touching (Hertenstein, Holmes, McCullough, \& Keltner, 2009; Hertenstein, Verkamp, Kerestes, \& Holmes, 2006). Therefore, understanding and employing gestures of touch make a profound contribution to creating and maintaining social relationships (Thayer, 1986). Furthermore, social psychology literature shows that gestures of touch have an impact on the attitudes and behaviors of another individual and that social touch can enhance a positive evaluation of the toucher (Crusco \& Wetzel, 1984; Fisher, Rytting, \& Heslin, 1976). It has recently been reported that interactions involving touch are considered more positive and exciting than are interactions with no touch (Schirmer et al., 2015), indicating that third party observers of touch may possibly share the touch recipient's social experiences. 
A possible mechanism that facilitates this experience of shared touch in a third-party observer is empathy. Empathy is a broad concept that pertains to an individual's reactions to the motor, perceptual or emotional state of another individual (Decety \& Jackson, 2006; Preston \& De Waal, 2002; ShamayTsoory, 2011). According to Davis (1983), empathy comprises four distinct measures: perspective taking, empathic concern, personal distress, and fantasy skills.

Albeit different in quality, all four of these measures relate to reactivity to the observed experiences of others, whether by taking the other's view and perspective, expressing concern or distress for another, or simply fantasizing being in another's place and situation. Interestingly, interindividual differences in empathic traits have been shown to influence the way in which individuals respond to social cues, recognize facial expressions, and even prefer interpersonal distance (Besel \& Yuille, 2010; Eisenberg \& Miller, 1987; Perry, Mankuta, \& Shamay-Tsoory, 2015).

In line with these findings, Lamm et al. (2015) have recently shown that neural networks activated during felt touch are also activated in the empathic observation of the same touch. Furthermore, Schaefer et al. (2012) found that the amount of neural activation for observed touch is correlated with levels of perspective taking, further demonstrating that levels of empathy may influence the way third-party observers are affected by social touch.

Interestingly, attenuation in the alphalmu rhythm $(8-13 \mathrm{~Hz})$ as measured by electroencephalography (EEG) has been found to relate to both felt and observed tactile stimuli. The alphalmu rhythm over central and occipital areas is attenuated both during the firsthand experience of tactile stimulation (Cheyne et al., 2003; Gaetz \& Cheyne, 2006) and during the observation of tactile stimulation (Coll et al., 2015; Perry, Bentin, Bartal, Lamm, \& Decety, 2010). This attenuation in the alphalmu rhythm, especially over central cortical areas (also called mu suppression), has been shown to correlate with blood-oxygen level-dependent (BOLD) signals in typical simulation mechanism areas such as the inferior parietal lobule, the dorsal premotor cortex, and the primary somatosensory cortex (Arnstein, Cui, Keysers, Maurits, \& Gazzola, 2011). Moreover, in the past few years, several studies have linked mu suppression to higher social information processing, including social skills (Oberman, Pineda, \& Ramachandran, 2007) and theory of mind (ToM; Perry, Stein, \& Bentin, 2011; Pineda \& Hecht, 2009). These studies and others indicate that mu suppression may underlie empathy (Cheng, Yang, Lin, Lee, \& Decety, 2008). Ultimately, we sought to explore the effect of interindividual differences in empathy levels on neural responses to observed social touch between two individuals. We predicted that empathy levels would modulate participants' behavioral and electrophysiological responses to photos of human social touch but not to photos of inanimate objects touching or photos of humans or objects without touch. We hypothesized that highly empathic individuals would rate photos of human social touch as more pleasant than would less empathic individuals and would rate these photos as more pleasant than the other three conditions. Furthermore, we hypothesized that highly empathic individuals would exhibit more mu suppression in observing photos of human social touch than would less empathic individuals and more mu suppression in observing social touch than nonsocial touch.

\section{Materials and method}

\section{Participants}

Participants included 54 undergraduate students (20 male) from the University of Haifa, Israel, who received course credit or payment in return for participating in the experiment. Participants ranged in age from 18 to 39 (mean=23.03, $S D$ $=4.09$ ). All participants were right-handed and reported normal or corrected-to-normal visual acuity. All participants had no history of psychiatric or neurological disorders, as confirmed by a screening interview. Two participants were omitted from the analyses because their behavioral scores were above three standard deviations from the average. Hence, the reported results are based on 52 participants (20 male). Written consent was obtained from each participant prior to the experiment, and the Ethical Review Committee of the University of Haifa approved the experiment.

\section{Assessment of empathy}

Following the EEG recordings, participants were asked to complete the Hebrew version (Even, 1993) of the Interpersonal Reactivity Index (IRI) questionnaire (Davis, 1983). This version has been translated into Hebrew and validated. The IRI is a 28 -item self-report measure consisting of four 7-item subscales, each tapping a different aspect of the global concept of empathy, broadly defined as a measure of reactivity to others. The four subscales are Perspective Taking (PT), which pertains to the tendency to adopt the psychological point of view of another; Fantasy Scale (FS), which pertains to the tendency of individuals to transpose themselves imaginatively into the feelings and actions of fictitious characters in books, movies, and plays; Empathic Concern (EC), which assesses feelings of sympathy and concern for unfortunate others; and Personal Distress (PD)w, which measures feeling of personal anxiety and unease in tense interpersonal settings. Participants were divided by the median averaged IRI score (3.5) into low IRI group (IRI $\leq 3.5 ; 25$ participants; mean IRI score $=3.15, S D=0.27$ ) and high IRI group (IRI $\leq 3.5 ; 27$ participants; mean IRI score $=3.8$, $S D=0.21$ ) for purposes of analysis. Following the median split analyses, correlation and multiple regressions analyses 
were conducted with each of the four subscales to examine the unique influence of each empathy construct to the responses for social touch observation.

\section{Stimuli task and design}

Participants sat approximately $60 \mathrm{~cm}$ from a CRT monitor and were presented with a computerized task (E-Prime 2.2, Psychological Software Tools, was used for stimulus presentation and experimental control). The participants were shown monochromatic photos of four different conditions: human touch, human nontouch, inanimate touch, and inanimate nontouch. The human touch condition contained photos depicting various types of social touch, including more reciprocal touch such as a hug, a handshake, or friendly hand holding and more nonreciprocal types of touch such as a tap on the shoulder (total of 65 photos; see Fig. 1). The inanimate touch condition included photos depicting two everyday objects (without any commercial logos) touching each other. The other two conditions contained the same humans or objects in proximity to one another but not touching (see Fig. 2). The combination of touch and object type therefore constituted a 2 $\times 2$ factorial design. Importantly, our study focused on social touch between dyads of heterosexual friends of the same gender. This criteria for selecting stimuli allowed us to avoid, as much as possible, the confounding influence of erotic touch that affects everyday interactions by producing a culturally dependent "erotic barrier" that prevents certain types of social touch (Heslin \& Alper, 1983). Therefore, we included only positive valence touch gestures because touch gestures between friends consist of mainly positive physical contacts of different objectives (greeting, parting, giving attention, helping, consoling, calming, and giving pleasure; see Suvilehto, Glerean, Dunbar, Hari, \& Nummenmaa, 2015). Thus, negative social touch gestures were excluded from our study. Interestingly, recent findings show that negative forms of touch, such as punishing, hurting, and scaring, are not relationship dependent and are rare among the types of social touch (Suvilehto et al., 2015). As a result, to the best of our knowledge our study is the first to address empathy for positive social situations, specifically social touch, compared to the vast literature on empathy for negative experiences.

Two dissimilar versions of the experiment were devised in order to differentiate between men's touch and women's touch and to reduce variability. Each gender responded to its congruent gender (i.e., men responded to social touch between men and women responded to social touch between women). Inanimate objects remained the same in the two experiments.

\section{Stimuli preparation}

The individuals in the photos either were photographed in a public environment during natural social interaction involving touch or were hired actors that performed the touch gestures on request. All of the social encounters presented were between same-sex friends who were not involved romantically or sexually. Models were asked to exhibit friendly social touch. Of 100 photos taken of social interaction containing touch, 65 photos were chosen for the experiment that clearly and intelligibly depicted the touch as rated by five independent raters. That is, the point of touch was clearly visible between the two individuals.

Consent was obtained from each model or actor in the photos prior to carrying out the experiments. Heads and faces were cropped out of the photos in order to simplify and reduce possible confounding effects.

\section{Procedure}

The stimuli were presented in five blocks of 52 trials each, for a total of 260 trials, in addition to four practice trials during which the experimenter made sure the participants understood

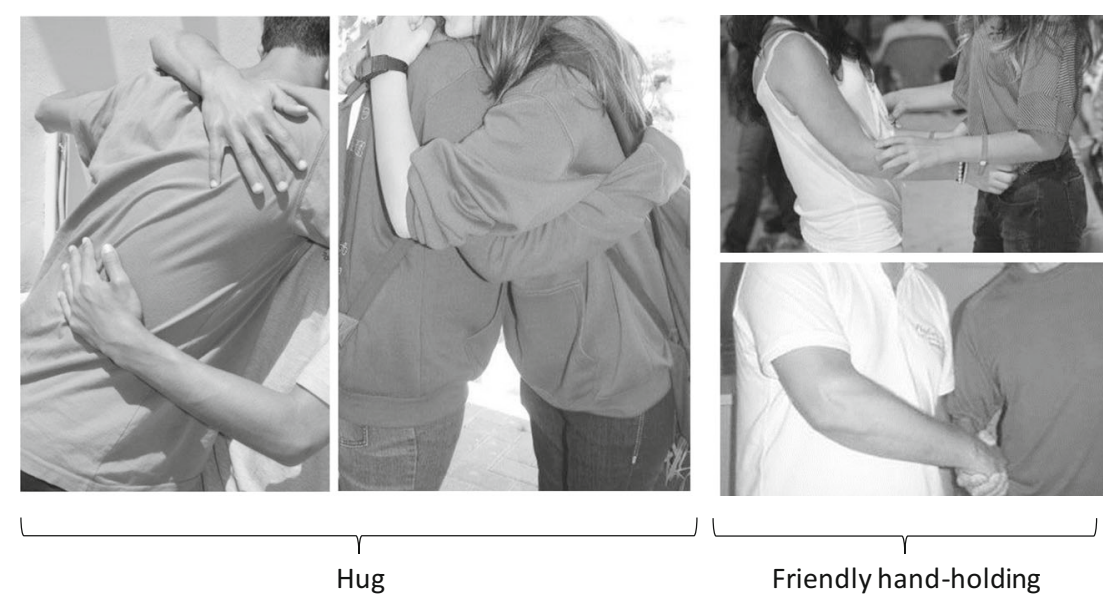

Fig. 1 Photos from the experimental condition of human touch depicting physical touch within a natural social interaction for both men and women 


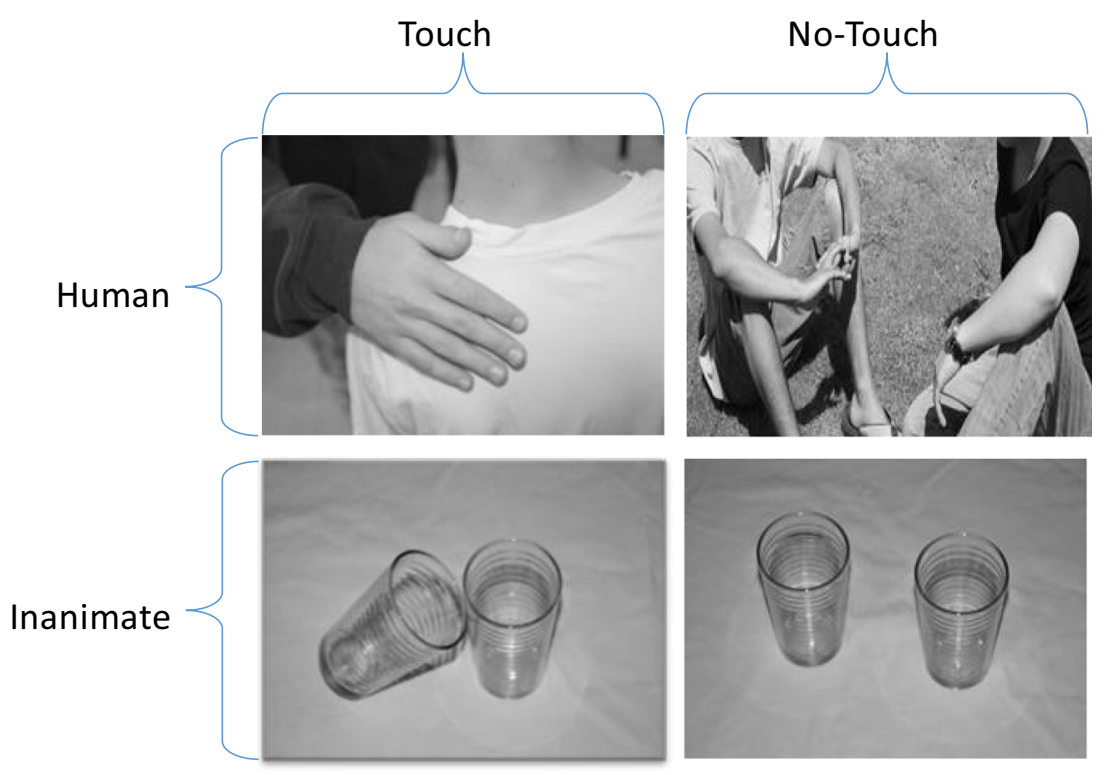

Fig. 2 Photos illustrating each of the four conditions explored in the study. The conditions can also be viewed according to two main categories of touch (with or without) and object present in the photo (human or inanimate)

the task. Each trial consisted of a fixation cross shown for 500 ms followed by a photograph presented for $1,300 \mathrm{~ms}$, followed by the ratings screen. Counterbalanced intertrial intervals of $200 \backslash 400 \backslash 600 \mathrm{~ms}$ were devised between each trial (see Fig. 3).

Responses required rating the extent of pleasant or unpleasant emotions elicited by the target. The ratings were made on a bipolar valence visual analog scale (VAS) which was later converted offline into numerical values that ranged from unpleasant feelings (-50) to pleasant feelings (50). According to IAPS protocol (Lang, Bradley, \& Cuthbert, 1998), participants were told the following: "At one extreme of the scale, you feel completely unpleasant, unhappy, annoyed, dissatisfied, melancholic or despaired. At the other end of the scale, you feel completely pleased, happy, satisfied, content or hopeful." Participants were further informed that a score of 0 at the scale's midpoint indicated a completely neutral state, neither a pleasant/happy nor an unpleasant/sad state.

\section{Data acquisition and analysis}

\section{EEG recording}

The EEG analog signals were recorded continuously (from $\mathrm{DC}$ with a low-pass filter set at $100 \mathrm{~Hz}$ ) by $32 \mathrm{Ag}-\mathrm{AgCl}$ intype active electrodes mounted on an elastic cap (Biosemi, http://www.biosemi.com/headcap.htm) according to the extended 10-20 system and from two additional electrodes placed at the right and left mastoids. All electrodes were referenced during recording to a common-mode signal (CMS) electrode between $\mathrm{C} 3$ and $\mathrm{Cz}$ and were subsequently rereferenced digitally (see Data processing section below). Eye movements as well as blinks were monitored using bipolar horizontal and vertical EOG derivations via two pairs of electrodes, with one pair attached to the external canthi and the other to the infraorbital and supraorbital regions of the right eye. Both EEG and EOG were digitally amplified and

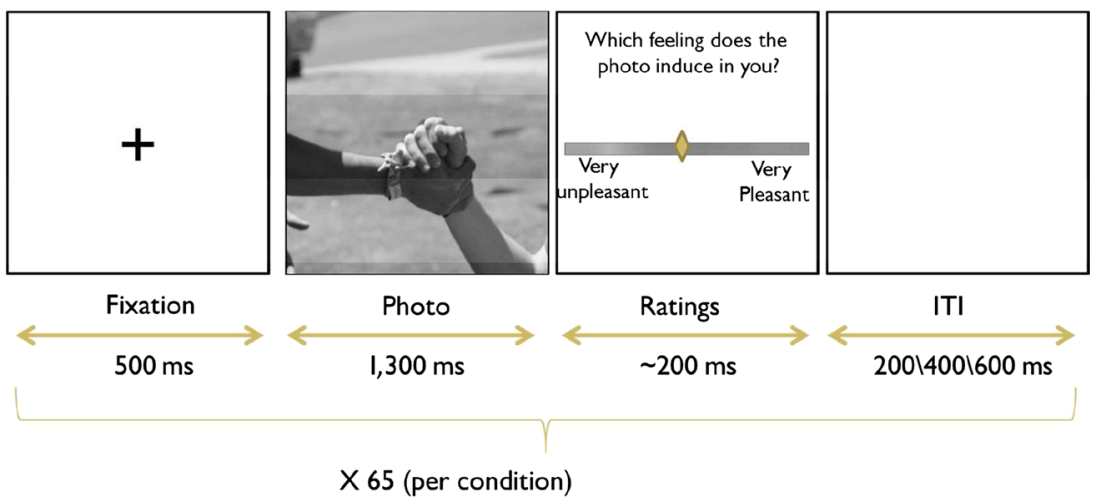

Fig. 3 Trial scheme in the computerized paradigm: A fixation cross followed by the photo and rating screen, with interstimulus interval between each trial. Each condition contained 65 photos that were counterbalanced between five blocks. ITI intertrial interval 
sampled at $512 \mathrm{~Hz}$ using a Biosemi Active II system (www. biosemi.com).

\section{Data processing}

Data were analyzed using Brain Vision Analyzer software 2.0 (Brain Products). Raw EEG data were initially $0.5 \mathrm{~Hz}$ highpass filtered $(24 \mathrm{~dB})$ and re-referenced off-line to the digital average of the two mastoids. An algorithm developed by Gratton, Coles, and Donchin, (1983) for off-line removal of ocular artifacts was used to correct for eye movements and blinks. Remaining artifacts exceeding $\pm 100 \mu \mathrm{V}$ in amplitude were rejected. Data were divided into 1,300-ms segments starting with each stimulus onset. A maximum of 10 trials were rejected for each participant in each condition.

Following previous studies that examined the role of mu suppression, sensorimotor resonance and empathy (Klimesch, 1999; Perry, Bentin, Shalev, et al., 2010; J. A. Pineda, 2005), we chose to focus on mu suppression in the lower range (8$10 \mathrm{~Hz}$ ).

For each such segment, the integrated power in the 8-10 $\mathrm{Hz}$ range was computed using a fast Fourier transform (FFT) performed at $0.5 \mathrm{~Hz}$ intervals (based on 2048 points per segment and using a Hanning window).

A suppression index was calculated as the logarithm of the ratio of the power during the experimental conditions relative to the power during the fixation point prior to presenting the photo and was used as the dependent variable (Perry, Bentin, Bartal, et al., 2010). The ratio (as opposed to simple subtraction) was used to control for the variability in absolute EEG power due to individual differences such as scalp thickness and electrode impedance (J. O. A. Pineda \& Oberman, 2006). Furthermore, because ratio data are inherently not normally distributed because of lower bounding, a log transform was also used to perform statistical analysis. Hence, a log ratio of less than zero indicates suppression in the EEG amplitude, whereas a value of zero indicates no change and values greater than zero indicate enhancement. Suppression was computed and analyzed at nine sites: occipital sites $\mathrm{O} 1, \mathrm{Oz}$, and $\mathrm{O} 2$, where classical alpha modulation is expected; central sites $\mathrm{C} 3, \mathrm{Cz}$, and $\mathrm{C} 4$, which are classical mu rhythm sites; and frontal sites F3, Fz, and F4 in order to investigate whether the effect is widespread or local.

\section{Results}

\section{Behavioral results}

A repeated-measures analysis was conducted for behavioral ratings, with touch (touch, no-touch) and object (human, inanimate) as within-subject factors and IRI score (low IRI, high IRI) as a between-subjects factor. There was a significant main effect for touch, $F(1,50)=46.897, p<.001$, such that photos containing touch were rated as inducing more pleasant emotions than photos that did not contain touch. There was also a significant main effect for object, $F(1,50)=44.693, p<.001$, such that photos containing humans were rated as inducing more pleasant emotions than photos containing inanimate objects. An interaction was found between Touch $\times$ Object, $F(1$, $50)=49.835, p<.001$. Follow-up $t$ tests showed that there was a significant difference between human and object in the touch condition but not in the nontouch condition, touch: $t(51)$ $=7.386, p<.001$, human touch $=14.02$, inanimate object touch $=2.6$; no-touch: $t(53)=1.073, p=.1 .03$, human notouch $=3.34$, inanimate object no-touch $=1.1$.

A significant second-order Touch $\times$ Object $\times$ IRI interaction was found, $F(1,50)=5.694, p<.05$. In order to further examine the second-order interaction, we conducted separate ANOVAs for humans and inanimate objects, with touch (touch, no-touch) and IRI (high IRI, low IRI) as within- and between-subject factors, respectively. In the human condition the ANOVA revealed significant interaction between touch and IRI, $F(1,50)=3.819, p<.05$. Planned $t$ tests showed that participants with higher IRI scores rated human touch as inducing more pleasant emotions than did participants with lower IRI scores. No differences were found between high and low IRI scores for human nontouch photos. In the inanimate object ANOVA, no interaction was found between IRI groups and touch $(p>.05)$ (see Fig. 4). No other main effect or interactions were found (all $p \mathrm{~s}>.05$ ).

To examine the differential contribution of each of the IRI subscales to observed touch, we examined simple correlations between the four subscales of the IRI and the human touch ratings. This analysis demonstrated a positive correlation between the PD subscale and human touch ratings $(r=0.239, p$ $<.05)$. The more PD, the more emotions elicited by observed human touch. The rest of the correlations were not significant.

In addition, we conducted a multiple regression analysis in order to decipher which IRI subscale is most prominent in predicting human touch ratings. The dependent variable was the human touch ratings and the independent predictors were the four subscales of the IRI: PT, FS, EC, and PD. The regression model was significant, $F(4,47)=$ $3.029, p<.05$. Five percent of the variance was explained by the four IRI subscales in the model. The subscale PD was found to be the only significant subscale predicting the ratings for human touch $(\beta=0.239, p<.05)$. The more PD the participant possesses, the higher the reported emotions that are elicited by observed human touch.

\section{EEG: 8-10-Hz mu suppression}

In order to determine whether mu suppression was primarily evident in regions above the somatosensory cortex, the suppression index was first analyzed using a repeated- 


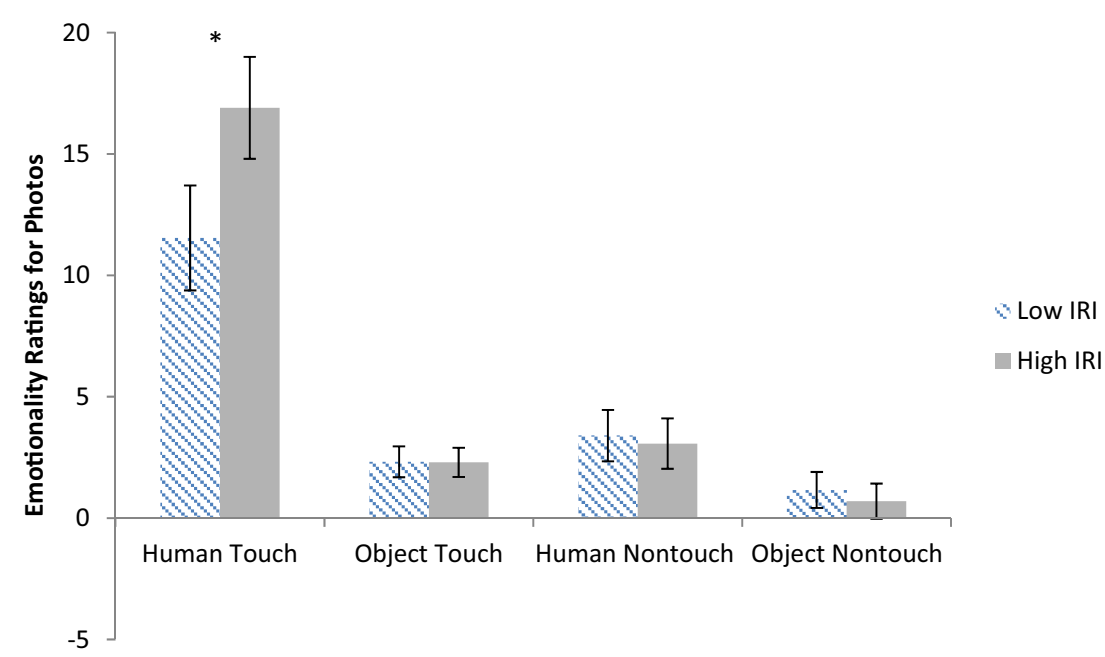

Fig. 4 Behavioral emotionality ratings for the high and low IRI groups. Participants with higher IRI scores rated human touch photos as inducing more pleasant emotions than did participants with lower IRI ratings. Error bars represent standart error of the mean. ${ }^{*} P<0.05$

measures ANOVA for the within-subjects factor of site (frontal, central, and occipital). A significant main effect of site showed that suppression differed significantly between the three sites, mean frontal $=-0.440$, mean central $=$ -0.487 , mean occipital $=-0.204 ; F(2,100)=102.355, p<$ .01. Pairwise comparisons revealed that suppression was significantly greater at the central sites as compared to all other sites $(p<.01$ for central vs. frontal and for central vs. occipital; all Bonferroni corrected). No other pairwise comparisons revealed significant differences.

Based on this distribution and on previous reports (Oberman et al., 2005; Perry \& Bentin, 2009), we focused on mu suppression at the central sites $\mathrm{C} 3$ and $\mathrm{C} 4$ that are particularly sensitive to sensorimotor areas. A suppression index (see the Data processing section) was analyzed using ANOVA with repeated measures. The factors were touch (touch, no-touch), object (human, inanimate), and hemisphere as within-subjects factors, and IRI (high, low) as a betweensubjects factor. There was a main effect of touch, $F(1,50)=$ $429.173, p<.001$, such that there was more mu suppression for photos containing touch than for those that did not contain touch. There was a main effect for object, $F(1,50)=418.575$, $p<.001$, such that there was more mu suppression for photos containing humans rather than objects. A significant secondorder interaction was found between touch, object, and IRI, $F(1,50)=3.457, p=.046$. In order to examine the secondorder interaction further, we conducted separate ANOVAs for humans and inanimate objects conditions, with touch (touch, no-touch) and hemisphere (left, right) as within-subjects factors and IRI (high, low) as between-subjects factors. In the human condition, ANOVA revealed a significant interaction between touch and IRI, $F(1,50)=4.69, p=.041$, in mu suppression. Planned $t$ tests showed that participants with higher IRI scores showed more mu suppression for human touch photos than did participants with lower IRI scores, $t(50)=1.99, p=.048$. No differences were found between high and low IRI scores for human nontouch photos. In the inanimate object condition, ANOVA showed no significant interaction between IRI groups and touch ( $p>.05$; Fig. 5).

\section{Confirmatory analyses for reciprocal photos}

In order to eliminate the possibility that our results were related to motor stimulation of the person performing the touch, we carried out additional analyses in which we excluded all the nonreciprocal photos and analyzed 36 photos in our experimental condition rather than 65 (see Fig. 1 to see example photos from these analyses). We randomly omitted 29 photos from each of the other three conditions in order to even all of the four conditions in the new analysis.

This analysis was performed on the same 52 participants, on electrodes $\mathrm{C} 3$ and $\mathrm{C} 4$, similar to the previous analysis. A suppression index (see Data processing section) was analyzed using ANOVA with repeated measures. The factors were touch (touch, no-touch), object (human, inanimate), and hemisphere as within-subjects factors, and IRI (high, low) as a between-subjects factor. The second-order interaction between touch, object, and IRI, $F(1,50)=8.020, p=0.007$, remained significant as before. We conducted separate ANOVAs for humans and inanimate objects conditions, with touch (touch, no-touch) and hemisphere (left, right) as withinsubjects factors and IRI (high, low) as between-subjects factors. The human condition ANOVA revealed a significant interaction between touch and IRI, $F(1,50)=7.541, p=$ .008 , in mu suppression. Planned $t$ tests showed again that participants with higher IRI scores showed more mu suppression for human touch photos than did participants with lower IRI scores, $t(50)=2.6147, p=.012$. No differences were found between high and low IRI scores for human nontouch 


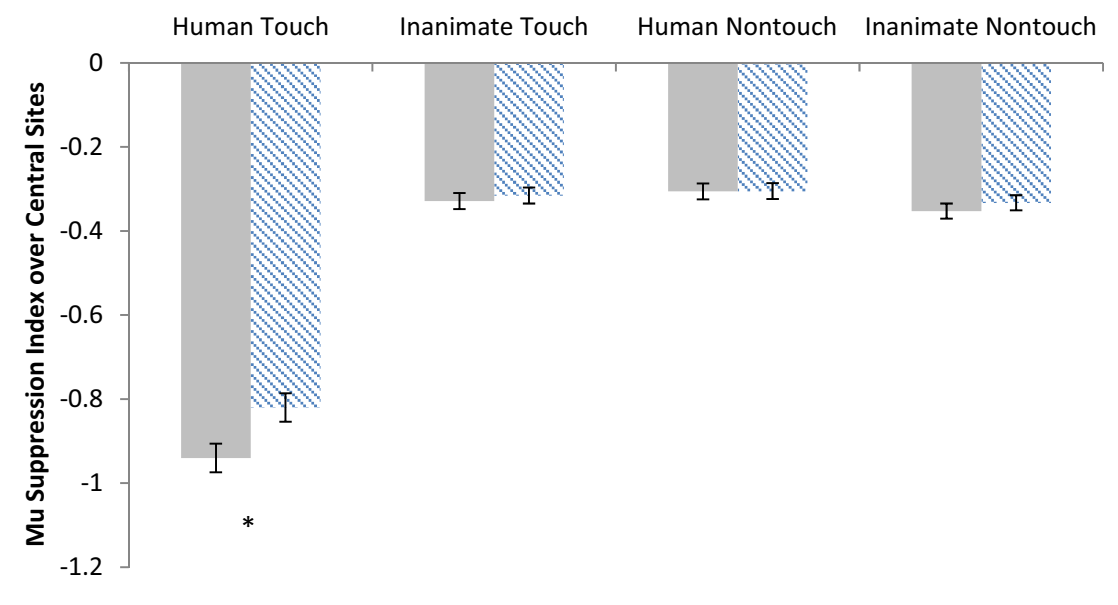

High IRI SLw IRI

Fig. 5 Suppression in the 8-10-Hz range over central sites between high and low IRI groups across all conditions. A significant difference was found between the groups for the human touch condition. Error bars represent standart error of the mean. $* P<0.05$

photos. The inanimate object condition ANOVA showed no significant interaction between IRI groups and touch $(p>.05)$.

\section{Brain and behavior}

Correlations between the behavioral ratings and the mu suppression amplitudes were calculated for electrodes $\mathrm{C} 3$ and $\mathrm{C} 4$ for each condition and were computed separately for the high and low IRI groups. For the high IRI group, a significant correlation was found for electrode $\mathrm{C} 4$ for the human touch condition, $r(27)=-0.307, \mathrm{p}=.049$, such that the more participants rated the human touch pictures as inducing pleasant emotions, the more mu suppression they exhibited in electrode C4 (see Fig. 6). No other correlations were found in the high IRI group and in the low IRI group.

\section{Multiple regressions for IRI subscales}

To examine the differential contribution of the IRI subscales, we carried out simple correlations between the four subscales

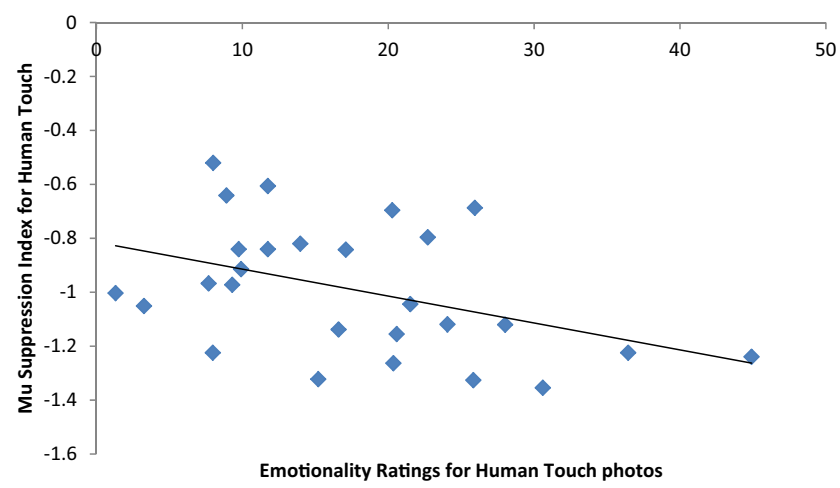

Fig. 6 The correlation between mu suppression in electrode $\mathrm{C} 4$ and emotionality ratings for human touch in the high IRI group $(r=-0.307$, $p<.05)$ of the IRI and the $\mathrm{C} 3 \mathrm{mu}$ suppression index. This analysis yielded a positive correlation between $\mathrm{PD}$ and $\mathrm{C} 3 \mathrm{mu}$ suppression for human touch $(r=0.233, p<.05)$. The more PD, the more mu suppression is elicited in electrode $\mathrm{C} 3$ in response to observed human touch. The rest of the correlations were not significant.

We used multiple regression analysis to determine which IRI subscale is most prominent in predicting mu suppression in electrode $\mathrm{C} 3$ for human touch. The dependent variable was the mu suppression index in electrode $\mathrm{C} 3$ for human touch and the independent predictors were the four subscales of the IRI: PT, FS, EC, and PD. The regression model was significant, $F(4,47)=2.335, \mathrm{p}<.05$. The four IRI subscales in the model explain $10 \%$ of the variance. The subscale PD was the most prominent in predicting the amount of mu suppression $(\beta=$ $0.399, p<.05)$ and PT was significant as well $(\beta=2.227, p<$ $.05)$. The more PD and perspective-taking skills the participant possesses, the more mu suppression is elicited for observed human touch.

In addition, simple correlations were examined between the four subscales of the IRI and the mu suppression index in electrode C4. A positive correlation was again found between PD and mu suppression C4 for human touch $(r=0.345$, $p<.05)$. The more $\mathrm{PD}$, the more mu suppression is elicited in $\mathrm{C} 4$ in response to observed human touch. No further correlations were found between the other subscales and $\mathrm{C} 4 \mathrm{mu}$ suppression.

We used multiple regression analysis to determine which IRI subscale is most prominent in predicting mu suppression in electrode $\mathrm{C} 4$ for human touch. The dependent variable was the mu suppression index in electrode $\mathrm{C} 4$ for human touch, and the independent predictors were the four subscales of the IRI: PT, FS, EC, and PD. The regression model was significant, $F(4,47)=3.475, p<.05$. The four IRI subscales in the model explain $15 \%$ of the variance. The subscale PD is the 
only prominent subscale predicting the amount of mu suppression $(\beta=0.549, p<.05)$ in the right hemisphere over sensory motor areas. The more PD the participant possesses, the more mu suppression is elicited for observed human touch.

Finally, to rule out the possibility that the findings were related to sex differences, we reanalyzed the behavioral and electrophysiological analyses with gender as a betweensubjects factor (20 males). No significant main effects were found for gender, and there were no interactions with gender (behavioral: all $F_{\mathrm{S}}<2.536$, all $p \mathrm{~s}>.118$; electrophysiological: all $F_{\mathrm{S}}<1.832$, all $\left.p \mathrm{~s}>.181\right)$.

\section{Discussion}

The main objective of this study was to examine whether interindividual differences in empathy modulated behavioral and electrophysiological responses to observed social interactions that involve touch. The behavioral results revealed that participants rated photos depicting human social touch as inducing more pleasant emotions than photos depicting nonsocial touch or nontouch. This finding shows that third-party observers of touch are affected by the social experience of touch observation and that social exchange of touch is deemed pleasant in the eye of the observer. Our study is in line with recent findings showing that photos containing touch are considered more positive and exciting compared to photos without touch (Schirmer et al., 2015) and further indicates that the perception of social touch can induce feelings in an observer who is not part of the touch itself.

Furthermore, as hypothesized, we found that highly empathic participants rated human social touch as inducing more pleasant feelings than did less empathic participants. Indeed, empathy is considered to play a crucial role in human social interactions and thus motivates prosocial behavior (Decety \& Michalska, 2010). Overall, it is possible that for less empathic individuals the emotion-sharing aspect was less pronounced because they are probably less motivated toward prosocial behavior, thus leading to lower ratings of the photos. It is important to note that this effect was not found for photos depicting nonsocial touch or nontouch, thus emphasizing the involvement of empathy specifically in social touch.

The EEG findings revealed that observation of human social touch induced more mu suppression in the $8-10 \mathrm{~Hz}$ at central sites than observation of nonsocial touch or no touch. Crucially, this suppression in response to human social touch was modulated by levels of empathy and, as hypothesized, participants with higher levels of empathy exhibited greater mu suppression for perception of social touch than did participants with lower levels of empathy.

Our results are in line with an accumulating body of literature on the involvement of mu suppression in the observation of tactile stimulation (Cheng et al., 2008; Coll et al., 2015;
Perry, Bentin, Bartal, et al., 2010) and show the involvement of mu suppression in a social situation. Past studies have shown that mu suppression occurs both while performing actions and while observing another performing actions (Oberman \& Ramachandran, 2007; Perry \& Bentin, 2009; Perry, Bentin, Bartal, et al., 2010; Perry, Stein, \& Bentin, 2011; J. A. Pineda, 2005; J. A. Pineda \& Hecht, 2009). These characteristics have led some researchers to tentatively link the suppression of mu rhythms with a human simulation system (J. A. Pineda, 2005) that enables simulation (and from this understanding) of the intentions, thoughts and feelings of another (Gallese, Keysers, \& Rizzolatti, 2004). The empathic trait levels reflect the extent to which a person is able to simulate and infer the intentions, thoughts, and feelings of another. Here we show that this trait also affects a person's neural response to a specific social aspect, namely, social touch. Empathy has been shown to correlate with neuronal activation in observing or listening to social scenes (Gazzola, AzizZadeh, \& Keysers, 2006; Hooker, Verosky, Germine, Knight, \& D'Esposito, 2010) and in observing nonsocial touch (Lamm et al., 2015; Osborn \& Derbyshire, 2010; Schaefer et al., 2012). Our study adds to this growing body of knowledge, demonstrating that empathy modulates a neuronal response to a social situation involving social touch. Hence, our results further substantiate the simulation network hypothesis for mu suppression.

For highly empathic participants, there was a significant correlation between the behavioral ratings and the electrophysiological measures for perception of human social touch, such that the more pleasant the emotions that participants experienced while observing the photos of social touch, the greater the mu suppression they exhibited while watching the photos. An important factor that may affect empathy is attention to social cues in another person (De Vignemont \& Singer, 2006; Singer \& Lamm, 2009). Interestingly, suppression in the lower alphalmu range $(8-10 \mathrm{~Hz})$ has been related to several attentional processes and to the amount of resources allocated to task performance (Klimesch, 1999; Perry, Bentin, Shalev, et al., 2010). Thus, it is possible that participants with lower empathy showed less mu suppression because they did not pay as much attention to the social touch stimuli as their highly empathic counterparts. In turn, this may explain why correlation between the behavioral ratings and the mu suppression power was significant only for highly empathic participants. Furthermore, this correlation was found only in the right hemisphere. Remarkably, lesions in the somatosensory cortex of the right hemisphere impaired patients' ability to recognize emotional facial expressions (Adolphs, Damasio, Tranel, Cooper, \& Damasio, 2000). Therefore, it is possible that the right hemisphere has a unique role in processing social touch.

We focused first on the IRI total scores because these scores provide a general measure of empathic abilities, 
whether from an emotional or a cognitive perspective. For instance, if an individual has moderate perspective-taking skills but high empathic concern and fantasy abilities, he or she can still have generally high empathic abilities.

In addition to our analyses with the IRI total averaged scores, we explored the unique contribution of each of the IRI subscales to the behavioral and electrophysiological responses to observed social touch. We found positive correlations between the IRI subscale PD and both the behavioral ratings for social touch and the mu suppression over somatosensory areas for social touch. Namely, the more PD an individual experiences, the more positively he or she deems human social touch and the more mu suppression he or she exhibits in response to social touch over sensorimotor regions. The PD scale measures a self-oriented motivated response and is defined as the tendency to experience distress or discomfort in response to extreme distress in others (Davis, 1994). Such self-oriented responses are primarily caused by thoughts concerning how individuals would feel in the same situations (Batson, Fultz, \& Schoenrade, 1987). These results are in line with Cheng et al. (2008) who found that mu suppression over somatosensory areas during the observation of hand actions, and not a moving dot, positively correlated with the PD subscale. The authors concluded that mu suppression is thus related to empathic mimicry. Our results also correspond with the findings reported by Yang, Decety, Lee, Chen, and Cheng (2009) who found that increased mu suppression was associated with increased PD in response to videos of individuals in possibly painful situations. Collectively, it may be suggested that increased mu suppression in the human touch condition is related to emotional contagion that occurs, apparently, even in positive situations of social touch such as hugging, hand holding, or affective touch to the upper body areas. Emotional contagion is an affective state that matches the other's emotional display (Decety \& Meyer, 2008). Indeed, mu rhythms were shown to be sensitive to affective influences and echoes sensorimotor processing (Pineda, 2005). Thus, apart from the overall empathic traits that play a crucial role in the response to social touch, PD that possibly reflects emotional contagion is a prominent benefactor to the neural and behavioral response to social touch.

It should be noted that the PD subscale of the IRI questionnaire is often regarded as a separate characteristic of empathy, unrelated to the other three factors in the questionnaire. Specifically, the psychometric analysis of Koller and Lamm (2015) indicates that the practical credit model (PCM), which is able to provide positive psychometric properties when it fits the data, does not hold for the PD subscale in the German IRI version (Koller \& Lamm, 2015). The same trend was also found in the Swedish version of the IRI, in which a general empathy factor underlies three of the IRI scales with the exception of PD (Cliffordson, 2002). In contrast to the German and Swedish versions, in the Chilean, Dutch, and Spanish versions of the IRI, the fantasy scale (FS) and not the PD was the only subscale that did not relate to the remaining three scales (De Corte et al., 2007; Escrivá, Navarro, \& García, 2004; Fernández, Dufey, \& Kramp, 2011). Furthermore, in the Chinese version of the IRI, a three-factor model was found with the FS and PD as different stand-alone factors, and the empathic concern and perspective-taking scales combined as the third factor (Siu, 2005). In the current study we used the Hebrew version of the IRI, which maintained its original fourfactor structure (Even, 1993) similar to that of the French version of the IRI (Gilet, Mella, Studer, Grühn, \& LabouvieVief, 2013). Future studies may address the discrepancies between the different psychometric properties of the IRI tool worldwide.

It is important to note that previous studies have reported cultural differences both in nonverbal communication in general (Elfenbein \& Ambady, 2002; Kita, 2009) and in physical contact in particular (Dibiase \& Gunnoe, 2004; Remland, Jones, \& Brinkman, 1995). In order to eliminate possible confounding effects related to cultural differences, the present study was carried out in one culture. Future studies may aim to address cultural differences and their effect on neural mechanisms for social touch.

Another important issue pertains to the nature of our stimuli in this experiment. The human conditions included photos that captured a moment in time of a dynamic social interaction that either contains physical touch or not. Different types of movements are inherent to social interactions, whether it is the hands that move in order to express and explain oneself, facial expressions, or different movements of the back and chin that are a part of a nonverbal body language (Henley, 1973). Touch is but one of the ways in which individuals express themselves within an interpersonal situation. In all of our photos, our participants may have inferred movement both with or without touch because the photos portray naturalistic social interactions that consist of all of the aforementioned movements. In order to partially eliminate the possibility that our results stemmed from motor simulation with the person performing the touch, we carried out additional analyses in which we analyzed only the reciprocal-touch photos, in which there is no clear toucher and recipient and both parties are touching and being touched. These analyses yielded the same results as before-namely, that during touch observation participants with higher empathic levels exhibit greater suppression in the mu band. These outcomes partially confirm that our results did not stem from direct motor simulation with either the toucher or the touched person but rather from the general experience of touch. Yet, an additional experimental condition of a person touching an inanimate object is needed, which will enable us to deduce whether the increase in mu suppression stems from motor stimulation with a toucher or from the general experience of touch observation. Future research might want the address this query. 
In addition, it is important to note that in the present experiment we did not include any human touch photos with nonmeaningful contact, because it was previously shown that the sight of a nonmeaningful, unintentional, or accidental touch decreased activation in the primary somatosensory cortex compared to meaningful, intentional touch. Moreover, the level of activation in these areas was correlated with the degree of intentionality of the touch (Ebisch et al., 2008). Therefore, we decided to omit such conditions in our experiment in order to focus on the response to meaningful social touch and its modulation by empathy traits. Moreover, our control human nontouch photos were taken immediately after the physical contact took place and as such convey a natural continuation of the social interaction. We thought this choice of control stimuli would reflect an ecological sequence of events and would enable us to decipher the added value of the physical contact in social situations. Our results showed that the physical contact increased the emotionality ratings of the photos compared to the social interactions without the touch. Therefore, we deduced that it was the physical contact itself, and not the social interaction, that elicited the higher behavioral ratings and the greater mu suppression. It would be interesting for future research to examine the response to observed nonverbal meaningful and emotional action of two bodies in a social interaction compared to neutral social interactions and isolate the effect of such meaningful, "contactless" action.

\section{Conclusion}

In conclusion, our study is the first to examine the relationship between empathy and social touch as reflected in attenuation of EEG oscillations in the mu band. Furthermore, our study is the first that addressed empathy for positive social events, specifically for social touch rather than for painful touch or aversive situations. It may be concluded that the perception of social touch is modulated by empathic levels in general and personal distress in particular. The current study further extends our knowledge regarding the magnitude of the role of empathy in everyday social interactions and the way interindividual differences in empathy levels influence both our conscious cognition and emotion and the automatic processes that take place in our brain.

\section{References}

Adolphs, R., Damasio, H., Tranel, D., Cooper, G., \& Damasio, A. R. (2000). A role for somatosensory cortices in the visual recognition of emotion as revealed by three-dimensional lesion mapping. The Journal of Neuroscience, 20(7), 2683-2690.
Arnstein, D., Cui, F., Keysers, C., Maurits, N. M., \& Gazzola, V. (2011). $\mu$-suppression during action observation and execution correlates with BOLD in dorsal premotor, inferior parietal, and SI cortices. The. Journal of Neuroscience, 31(40), 14243-14249.

Batson, C. D., Fultz, J., \& Schoenrade, P. A. (1987). Distress and empathy: Two qualitatively distinct vicarious emotions with different motivational consequences. Journal of Personality, 55(1), 19-39.

Besel, L. D. S., \& Yuille, J. C. (2010). Individual differences in empathy: The role of facial expression recognition. Personality and Individual Differences, 49(2), 107-112.

Blakemore, S.-J. S.-J., Bristow, D., Bird, G., Frith, C., \& Ward, J. (2005). Somatosensory activations during the observation of touch and a case of vision-touch synaesthesia. Brain, 128(7), 1571-1583.

Bolognini, N., Rossetti, A., Convento, S., \& Vallar, G. (2013). Understanding others' feelings: The role of the right primary somatosensory cortex in encoding the affective valence of others' touch. The Journal of Neuroscience, 33(9), 4201-4205.

Bufalari, I., Aprile, T., Avenanti, A., Di Russo, F., \& Aglioti, S. M. (2007). Empathy for pain and touch in the human somatosensory cortex. Cerebral Cortex, 17(11), 2553-2561.

Cheng, Y., Yang, C.-Y., Lin, C.-P., Lee, P.-L., \& Decety, J. (2008). The perception of pain in others suppresses somatosensory oscillations: A magnetoencephalography study. NeuroImage, 40(4), 1833-1840.

Cheyne, D., Gaetz, W., Garnero, L., Lachaux, J. P., Ducorps, A., Schwartz, D., \& Varela, F. J. (2003). Neuromagnetic imaging of cortical oscillations accompanying tactile stimulation. Cognitive Brain Research, 17(3), 599-611.

Cliffordson, C. (2002). The hierarchical structure of empathy: Dimensional organization and relations to social functioning. Scandinavian Journal of Psychology, 43(1), 49-59.

Coll, M.-P., Bird, G., Catmur, C., \& Press, C. (2015). Cross-modal repetition effects in the mu rhythm indicate tactile mirroring during action observation. Cortex, 63, 121-131.

Crusco, A. H., \& Wetzel, C. G. (1984). The midas touch the effects of interpersonal touch on restaurant tipping. Personality and Social Psychology Bulletin, 10(4), 512-517.

Davis, M. H. (1983). Measuring individual differences in empathy: Evidence for a multidimensional approach. Journal of Personality and Social Psychology, 44(1), 113.

Davis, M. H. (1994). Empathy: A social psychological approach. Boulder: Westview Press.

De Corte, K., Buysse, A., Verhofstadt, L. L., Roeyers, H., Ponnet, K., \& Davis, M. H. (2007). Measuring empathic tendencies: Reliability and validity of the Dutch version of the interpersonal reactivity index. Psychologica Belgica, 47(4), 235.

De Vignemont, F., \& Singer, T. (2006). The empathic brain: how, when and why? Trends in Cognitive Sciences, 10(10), 435-441.

Decety, J., \& Jackson, P. L. (2006). A social-neuroscience perspective on empathy. Current Directions in Psychological Science, 15(2), 54 58.

Decety, J., \& Meyer, M. (2008). From emotion resonance to empathic understanding: A social developmental neuroscience account. Development and Psychopathology, 20(04), 1053.

Decety, J., \& Michalska, K. J. (2010). Neurodevelopmental changes in the circuits underlying empathy and sympathy from childhood to adulthood. Developmental Science, 13(6), 886-899.

Dibiase, R., \& Gunnoe, J. (2004). Gender and culture differences in touching behavior. The Journal of Social Psychology, 144(1), 4962.

Ebisch, S. J. H., Perrucci, M. G., Ferretti, A., Del Gratta, C., Romani, G. L., \& Gallese, V. (2008). The sense of touch: Embodied simulation in a visuotactile mirroring mechanism for observed animate or inanimate touch. Journal of Cognitive Neuroscience, 20(9), 16111623.

Eisenberg, N., \& Miller, P. A. (1987). The relation of empathy to prosocial and related behaviors. Psychological Bulletin, 101(1), 91. 
Elfenbein, H. A., \& Ambady, N. (2002). On the universality and cultural specificity of emotion recognition: a meta-analysis. Psychological Bulletin, 128(2), 203.

Escrivá, V. M., Navarro, M. D. F., \& García, P. S. (2004). La medida de la empatía: Análisis del Interpersonal Reactivity Index. [Measuring empathy: The Interpersonal Reactivity Index.]. Psicothema, 16(2), 255-260.

Even, A. (1993). Effects of differentiation and tenure on sympathy, empathy and burnout (Unpublished doctoral dissertation, Bar Ilan University, Ramat Gan, Israel).

Fernández, A. M., Dufey, M., \& Kramp, U. (2011). Testing the psychometric properties of the Interpersonal Reactivity Index (IRI) in Chile. European Journal of Psychological Assessment, 27(3), 179-185.

Fisher, J. D., Rytting, M., \& Heslin, R. (1976). Hands touching hands: Affective and evaluative effects of an interpersonal touch. Sociometry, 39(4), 416-421.

Gaetz, W., \& Cheyne, D. (2006). Localization of sensorimotor cortical rhythms induced by tactile stimulation using spatially filtered MEG. NeuroImage, 30(3), 899-908.

Gallese, V., Keysers, C., \& Rizzolatti, G. (2004). A unifying view of the basis of social cognition. Trends in Cognitive Sciences, 8(9), 396403

Gazzola, V., Aziz-Zadeh, L., \& Keysers, C. (2006). Empathy and the somatotopic auditory mirror system in humans. Current Biology, 16(18), 1824-1829.

Gilet, A. L., Mella, N., Studer, J., Grühn, D., \& Labouvie-Vief, G. (2013). Assessing dispositional empathy in adults: A French validation of the Interpersonal Reactivity Index (IRI). Canadian Journal of Behavioural Science/Revue canadienne des sciences $d u$ comportement, 45(1), 42.

Gratton, G., Coles, M. G. H., \& Donchin, E. (1983). A new method for off-line removal of ocular artifact. Electroencephalography and Clinical Neurophysiology, 55(4), 468-484.

Henley, N. M. (1973). Power, sex, and nonverbal communication. Berkeley Journal of Sociology, 18, 1-26.

Hertenstein, M. J., Verkamp, J. M., Kerestes, A. M., \& Holmes, R. M. (2006). The communicative functions of touch in humans, nonhuman primates, and rats: A review and synthesis of the empirical research. Genetic, Social, and General Psychology Monographs, 132(1), 5-94.

Hertenstein, M. J., Holmes, R., McCullough, M., \& Keltner, D. (2009). The communication of emotion via touch. Emotion, 9(4), 566.

Heslin, R., \& Alper, T. (1983) Touch: A bonding gesture. In J. M. Wiemann, \& R. P. Harrison (Eds.), Non verbal interaction (pp 47 75). Beverly Hills: Sage.

Hooker, C. I., Verosky, S. C., Germine, L. T., Knight, R. T., \& D’Esposito, M. (2010). Neural activity during social signal perception correlates with self-reported empathy. Brain Research, 1308, $100-113$.

Keysers, C., Wicker, B., Gazzola, V., Anton, J.-L., Fogassi, L., \& Gallese, V. (2004). A touching sight: SII/PV activation during the observation and experience of touch. Neuron, 42(2), 335-346.

Kita, S. (2009). Cross-cultural variation of speech-accompanying gesture: A review. Language and Cognitive Processes, 24(2), 145-167.

Klimesch, W. (1999). EEG alpha and theta oscillations reflect cognitive and memory performance: a review and analysis. Brain Research Reviews, 29(2), 169-195.

Koller, I., \& Lamm, C. (2015). Item response model investigation of the (German) Interpersonal Reactivity Index Empathy Questionnaire. European Journal of Psychological Assessment, 31(3), 211-221.

Lamm, C., Silani, G., \& Singer, T. (2015). Distinct neural networks underlying empathy for pleasant and unpleasant touch. Cortex, 70, 79 89.
Lang, P. J., Bradley, M. M., \& Cuthbert, B. N. (1998). Emotion, motivation, and anxiety: Brain mechanisms and psychophysiology. Biological Psychiatry, 44(12), 1248-1263.

Oberman, L. M., \& Ramachandran, V. S. (2007). The simulating social mind: the role of the mirror neuron system and simulation in the social and communicative deficits of autism spectrum disorders. Psychological Bulletin, 133(2), 310.

Oberman, L. M., Hubbard, E. M., McCleery, J. P., Altschuler, E. L., Ramachandran, V. S., \& Pineda, J. A. (2005). EEG evidence for mirror neuron dysfunction in autism spectrum disorders.Cognitive. Brain Research, 24(2), 190-198.

Oberman, L. M., Pineda, J. A., \& Ramachandran, V. S. (2007). The human mirror neuron system: A link between action observation and social skills. Social Cognitive and Affective Neuroscience, $2(1), 62-66$.

Osborn, J., \& Derbyshire, S. W. (2010). Pain sensation evoked by observing injury in others. Pain, 148(2), 268-274.

Perry, A., \& Bentin, S. (2009). Mirror activity in the human brain while observing hand movements: A comparison between EEG desynchronization in the $\mu$-range and previous fMRI results. Brain Research, 1282, 126-132.

Perry, A., Bentin, S., Bartal, I. B.-A., Lamm, C., \& Decety, J. (2010). "Feeling" the pain of those who are different from us: Modulation of EEG in the mu/alpha range. Cognitive, Affective, \& Behavioral Neuroscience, 10(4), 493-504.

Perry, A., Bentin, S., Shalev, I., Israel, S., Uzefovsky, F., Bar-On, D., \& Ebstein, R. P. (2010). Intranasal oxytocin modulates EEG mu/alpha and beta rhythms during perception of biological motion. Psychoneuroendocrinology, 35(10), 1446-1453.

Perry, A., Stein, L., \& Bentin, S. (2011). Motor and attentional mechanisms involved in social interaction-Evidence from mu and alpha EEG suppression. NeuroImage, 58(3), 895-904.

Perry, A., Mankuta, D., \& Shamay-Tsoory, S. G. (2015). OT promotes closer interpersonal distance among highly empathic individuals. Social Cognitive and Affective Neuroscience, 10(1), 3-9.

Pineda, J. A. (2005). The functional significance of mu rhythms: Translating "seeing" and "hearing" into "doing.". Brain Research Reviews, 50(1), 57-68.

Pineda, J. A., \& Hecht, E. (2009). Mirroring and mu rhythm involvement in social cognition: Are there dissociable subcomponents of theory of mind? Biological Psychology, 80(3), 306314.

Pineda, J. O. A., \& Oberman, L. M. (2006). What goads cigarette smokers to smoke? Neural adaptation and the mirror neuron system. Brain Research, 1121(1), 128-135.

Preston, S. D., \& De Waal, F. (2002). Empathy: Its ultimate and proximate bases. Behavioral and Brain Sciences, 25(01), 120.

Remland, M. S., Jones, T. S., \& Brinkman, H. (1995). Interpersonal distance, body orientation, and touch: Effects of culture, gender, and age. The Journal of Social Psychology, 135(3), 281-297.

Rizzolatti, G., Fogassi, L., \& Gallese, V. (2001). Neurophysiological mechanisms underlying the understanding and imitation of action. Nature Reviews Neuroscience, 2(9), 661-670.

Rossetti, A., Miniussi, C., Maravita, A., \& Bolognini, N. (2012). Visual perception of bodily interactions in the primary somatosensory cortex. European Journal of Neuroscience, 36(3), 2317-2323.

Schaefer, M., Heinze, H.-J., \& Rotte, M. (2005). Seeing the hand being touched modulates the primary somatosensory cortex. Neuroreport, 16(10), 1101-1105.

Schaefer, M., Xu, B., Flor, H., \& Cohen, L. G. (2009). Effects of different viewing perspectives on somatosensory activations during observation of touch. Human Brain Mapping, 30(9), $2722-2730$ 
Schaefer, M., Heinze, H.-J., \& Rotte, M. (2012). Embodied empathy for tactile events: Interindividual differences and vicarious somatosensory responses during touch observation. NeuroImage, 60(2), 952957.

Schirmer, A., Reece, C., Zhao, C., Ng, E., Wu, E., \& Yen, S. C. (2015). Reach out to one and you reach out to many: Social touch affects third-party observers. British Journal of Psychology, 106(1), 107132.

Shamay-Tsoory, S. G. (2011). The neural bases for empathy. The Neuroscientist, 17(1), 18-24.

Singer, T., \& Lamm, C. (2009). The social neuroscience of empathy. Annals of the New York Academy of Sciences, 1156(1), 81-96.
Siu, A. M. H. (2005). Validation of the Interpersonal Reactivity Index in a Chinese context. Research on Social Work Practice, 15(2), 118126.

Suvilehto, J. T., Glerean, E., Dunbar, R. I., Hari, R., \& Nummenmaa, L. (2015). Topography of social touching depends on emotional bonds between humans. Proceedings of the National Academy of Sciences, 201519231.

Thayer, S. (1986). History and strategies of research on social touch. Journal of Nonverbal Behavior, 10(1), 12-28.

Yang, C.-Y., Decety, J., Lee, S., Chen, C., \& Cheng, Y. (2009). Gender differences in the mu rhythm during empathy for pain: An electroencephalographic study. Brain Research, 1251, 176-184. 